PROCEEDINGS OF THE

AMERICAN MATHEMATICAL SOCIETY

Volume 127, Number 4, April 1999, Pages 1215-1220

S 0002-9939(99)04721-8

\title{
RECURRENT CRITICAL POINTS AND TYPICAL LIMIT SETS OF RATIONAL MAPS
}

\author{
ALEXANDER M. BLOKH, JOHN C. MAYER, AND LEX G. OVERSTEEGEN \\ (Communicated by Mary Rees)
}

\begin{abstract}
We consider a rational map $f: \widehat{\mathbb{C}} \rightarrow \widehat{\mathbb{C}}$ of the Riemann sphere with normalized Lebesgue measure $\mu$ and show that if there is a subset of the Julia set $J(f)$ of positive $\mu$-measure whose points have limit sets not contained in the union of the limit sets of recurrent critical points, then $\omega(x)=\widehat{\mathbb{C}}=J(f)$ for $\mu$-a.e. point $x$ and $f$ is conservative, ergodic and exact.
\end{abstract}

\section{INTRODUCTION}

Let $f: \widehat{\mathbb{C}} \rightarrow \widehat{\mathbb{C}}$ be a rational map of the Riemann sphere and let $\mu$ denote normalized Lebesgue measure. We study the limit sets (by which we mean $\omega$-limit sets) of points for $f$. By Sullivan's Classification Theorem the limit set of a point in the Fatou set is either an attractive or parabolic periodic orbit, or a simple closed curve. On the other hand, Lyubich proved in [Lyu83] that for rational maps $f$ such that $J(f)$ is not the entire sphere the limit set of $\mu$-a.e. point in $J(f)$ is contained in the postcritical set (the union of limit sets of all critical points). Later McMullen showed [McM94, page 42] that either the limit set of $\mu$-a.e. point in $J(f)$ is contained in the postcritical set, or $J(f)$ is the entire sphere and $f$ is ergodic. Julia Barnes recently strengthened this theorem by showing that in the second case the map is exact and conservative.

Thus, it is already known that for a rational map $f$ either the limit set of $\mu$-a.e. point in $J(f)$ is contained in the postcritical set, or $J(f)$ is the entire sphere and the map $f$ is conservative, ergodic and exact. Our aim is to replace the postcritical set here with the union of limit sets of all recurrent critical points.

\section{MAIN THEOREM}

A rational map $f$ is called conservative if for all sets $X$ with $\mu(X)>0$, there exists a $k>0$ such that $\mu\left(f^{-k}(X) \cap X\right)>0$. It is called ergodic if $\mu(X) \in\{0,1\}$ whenever $f^{-1}(X)=X$, and exact if $\mu(X) \in\{0,1\}$ whenever $f^{-n} \circ f^{n}(X)=$ $X$ for all $n>0$ (by Rohlin [Roh64], for a certain class of mappings, including

Received by the editors July 23, 1997.

1991 Mathematics Subject Classification. Primary 58F23, 28D99; Secondary 34C35.

Key words and phrases. Julia set, complex analytic dynamics, limit set, recurrent critical point.

The first author was partially supported by NSF Grant DMS 9626303 and the third author by NSF Grant DMS 9704903. Portions of this paper were presented by the third author at the Spring Topology Conference in Lafayette, Louisiana, in April, 1997.

(C)1999 American Mathematical Society 
rational maps, this definition is equivalent to the traditional one according to which $\bigcap_{n \geq 0} f^{-n}(\mathcal{B})$ contains only sets of measure 0 or 1 for the $\sigma$-algebra $\mathcal{B}$ of Borel sets). Clearly, all exact rational maps are ergodic.

Our main result is the following:

Theorem 2.1. Let $f$ be a rational map on the sphere, $C$ the set of all its recurrent critical points and

$$
A_{f}=\bigcup_{c \in C} \omega(c)
$$

Then exactly one of the following holds:

1. $\mu(J(f))=0$, and for $\mu$-a.e. point $z$ on the sphere $\omega(z)$ is either a simple closed curve or an attractive or parabolic periodic orbit,

2. $\mu(J(f))>0$ and $\omega(z) \subset A_{f}$ for $\mu$-a.e. point $z \in J(f)$, or

3. the set of points $z \in J(f)$ such that $\omega(z) \not \subset A_{f}$ has positive $\mu$-measure, $f$ is exact, ergodic and conservative, and $\omega(z)=\widehat{\mathbb{C}}=J(f)$ for $\mu$-a.e. point $z \in \widehat{\mathbb{C}}$.

As we mention in the Introduction, Theorem 2.1 extends previously known results with the set $A_{f}$ replacing the postcritical set. Let us state an easy corollary of Theorem 2.1 .

Corollary 2.2. Let $f$ be a rational map with no recurrent non-periodic critical points. Then either 1) $\mu(J(f))=0$ or 2) $\omega(z)=\widehat{\mathbb{C}}=J(f)$ for $\mu$-a.e. point $z \in \widehat{\mathbb{C}}$ and $f$ is exact, ergodic and conservative.

Observe that the limit set of a non-recurrent critical point $c$ of $f$ may contain other critical points, but cannot contain $c$. Urbański [Urb94] proves stronger results about the Julia set under the assumption that the Julia set is not the entire sphere and $f$ is a rational map without non-periodic recurrent critical points.

Some tools used in the proof of Theorem 2.1 were obtained by Mañé [Mn93] and Barnes [Bar97]. An important result of Mañé [Mn93, Theorem II] deals with backward dynamics of rational functions and can be stated as follows:

Theorem 2.3 (Mañé). Suppose that $f$ is a rational map of the sphere and $x \notin A_{f}$ is not a parabolic periodic point. Then for all $\varepsilon>0$ there exists a neighborhood $W$ of $x$ such that the following hold:

1. for all $n \geq 0$, every connected component of $f^{-n}(W)$ has diameter at most $\varepsilon$,

2. there exists $N$ such that for any connected component $U$ of $f^{-n}(W)$ the degree of $f^{n} \mid U$ is at most $N$,

3. for any $\varepsilon_{1}>0$ there exists $k$ such that every connected component of $f^{-n}(W)$ has diameter less than $\varepsilon_{1}$ provided that $n>k$.

The result by Julia Barnes [Bar97, Theorem 3.6] allows us to prove exactness in part 3 of Theorem 2.1. Following Barnes, a set $X \subset \widehat{\mathbb{C}}$ with $\mu(X)>0$ is lim sup full if $\lim \sup \mu\left(f^{n}(X)\right)=1$. The map $f$ is lim sup full if every set of positive measure is lim sup full.

Theorem 2.4 (Barnes). Let $f$ be a lim sup full rational map of the sphere. Then $f$ is conservative and exact. 


\section{BOUNDED DISTORTION}

For the sake of convenience sets homeomorphic to the unit disk are called simply disks. By an $\varepsilon$-disk we mean a disk of radius $\varepsilon$ and by a round disk we mean an $\varepsilon$-disk for some $\varepsilon>0$.

We prove a distortion lemma from complex analysis. Although in Theorem 2.1 we deal with normalized Lebesgue measure $\mu$ it is convenient to use also plane Lebesgue measure $\lambda$. Clearly, a lot of objects (such as sets of zero measure, points of density of sets and the like) are the same for both measures and we will rely upon this freely throughout the paper. Since our distortion lemma allows critical points it makes better sense to consider quotients of measures of sets rather than quotients of derivatives. We will need some definitions. By the relative $\lambda-($ or $\mu-)$ measure of the set $X$ in the set $Y$ we understand the quotient $\lambda(X \cap Y) / \lambda(Y)$ (or $\mu(X \cap Y) / \mu(Y)$, respectively).

Lemma 3.1. Let $V \subset W$ be round concentric disks such that $\bmod (W \backslash V)=m \geq$ $4 \pi k$, and $g: U \rightarrow W$ an analytic $k$-to-1 covering map of a disk $U$ onto $W$ with no critical values of $g$ on $\partial V$.

Let $V^{\prime}$ be a component of $g^{-1}(V)$. Then $V^{\prime}$ is a disk, $\bmod \left(U \backslash V^{\prime}\right) \geq m / k$, and if $\delta=\operatorname{diam}\left(V^{\prime}\right)$, then for any set $A \subset V$ with the relative $\lambda$-measure of $A$ in $V$ at least $\varepsilon$, the relative measure of $g^{-1}(A)$ in the $\delta$-disk centered at any point of $V^{\prime}$ is at least $10^{-9} e^{-4 \pi m} \varepsilon \frac{\lambda(V)}{\lambda(W)}$.

Proof. We prove the lemma in two steps. First we show that the relative $\lambda$-measure of $g^{-1}(A)$ in $V^{\prime}$ is at least $10^{-4} e^{-4 \pi m} \varepsilon$. Then we prove that the relative $\lambda$-measure of $V^{\prime}$ inside any disk of radius $\delta$ is at least $10^{-5} \lambda(V) / \lambda(W)$. Together these two statements imply the lemma. The main idea of the proof is to normalize the map by means of conformal isomorphisms and then apply the Koebe lemma and well-known inequalities from the theory of analytic functions.

Let $\mathbb{D}$ be the unit disk with the center at 0 . We may assume that $W=\mathbb{D}$ and $V$ is an $r$-disk with the center at 0 and $r=e^{-2 \pi m}$ (the last equation is easily computed from the fact that $\bmod (\mathbb{D} \backslash V)=m)$. Let $V^{\prime}$ be a component of $g^{-1}(V)$. Since $\partial V$ contains no critical values, $V^{\prime}$ is a disk. Choose a point $x \in V^{\prime}$ so that $g(x)=0$. Let also $\phi: U \rightarrow \mathbb{D}$ be a conformal isomorphism such that $\phi(x)=0$. Let $A \subset V$ be a subset of relative $\lambda$-measure at least $\varepsilon$.

The assumptions of the lemma and properties of moduli easily imply that

$$
\bmod \left(U \backslash V^{\prime}\right) \geq m / k \text {. }
$$

Since $\phi$ is a conformal isomorphism we see that $\bmod \left(\mathbb{D} \backslash \phi\left(V^{\prime}\right)\right) \geq m / k$ too. By a well-known inequality [Mil92]

$$
\operatorname{diam}\left(\phi\left(V^{\prime}\right)\right) \leq \sqrt{\lambda(\mathbb{D}) / \bmod \left(\mathbb{D} \backslash \phi\left(V^{\prime}\right)\right)} \leq \sqrt{k \pi / m} \leq 1 / 2 .
$$

Since $0 \in \phi\left(V^{\prime}\right)$ we conclude that the set $\phi\left(V^{\prime}\right)$ is contained in the $\frac{1}{2}$-disk $V^{\prime \prime}$ with center at 0 .

Consider the map $G=g \circ \phi^{-1}: \mathbb{D} \rightarrow \mathbb{D}$ which maps $\phi\left(V^{\prime}\right)$ onto $V$. Since $\phi\left(V^{\prime}\right) \subset V^{\prime \prime}$, by the Schwarz-Pick lemma [CG93, p. 13] for any $z \in \phi\left(V^{\prime}\right)$ we have

$$
\left|G^{\prime}(z)\right| \leq \frac{1-|G(z)|^{2}}{1-|z|^{2}} \leq 4 / 3
$$


The $\lambda$-measure of the set $A$ is at least $\varepsilon \lambda(V)=\varepsilon \pi r^{2}$. Since

$$
\varepsilon \pi r^{2}=\varepsilon \lambda(V) \leq \lambda(A) \leq \int_{G^{-1}(A) \cap \phi\left(V^{\prime}\right)}\left|G^{\prime}\right|^{2} d \lambda,
$$

it follows that $\lambda\left(G^{-1}(A) \cap \phi\left(V^{\prime}\right)\right) \geq(9 / 16) \varepsilon \pi r^{2}=(9 / 16) \varepsilon \pi e^{-4 \pi m}$. On the other hand $\lambda\left(\phi\left(V^{\prime}\right)\right) \leq \lambda\left(V^{\prime \prime}\right)=\pi / 4$. We conclude that $G^{-1}(A) \cap \phi\left(V^{\prime}\right)$ has relative $\lambda$-measure at least $(9 / 4) \varepsilon e^{-4 \pi m}$ in $\phi\left(V^{\prime}\right)$. Let $l=\min \left\{\left|\phi^{\prime}(x)\right|: \phi(x) \in V^{\prime \prime}\right\}$ and $L=\max \left\{\left|\phi^{\prime}(x)\right|: \phi(x) \in V^{\prime \prime}\right\}$. Since $\phi\left(V^{\prime}\right) \subset V^{\prime \prime}$, then by the Koebe Lemma [CG93] for any $x, y \in V^{\prime}$ we have

$$
\left|\phi^{\prime}(x) / \phi^{\prime}(y)\right| \leq L / l \leq\left(\frac{1+1 / 2}{1-1 / 2}\right)^{4}=81 .
$$

This implies that the relative measure of $g^{-1}(A)$ in $V^{\prime}$ is

$$
\frac{\lambda\left(g^{-1}(A) \cap V^{\prime}\right)}{\lambda\left(V^{\prime}\right)} \geq(9 / 4) \varepsilon e^{-4 \pi m} / 81^{2} \geq 10^{-4} \varepsilon e^{-4 \pi m} .
$$

Above we showed that $\left|G^{\prime}(z)\right| \leq 4 / 3$ for $z \in \phi\left(V^{\prime}\right)$. By the same argument as above, this implies that $\lambda\left(\phi\left(V^{\prime}\right)\right) \geq 9 / 16 \cdot \lambda(V)$. On the other hand if $\delta=\operatorname{diam}\left(V^{\prime}\right)$, then $\delta \leq 1 / l$. Since $L \leq 81 l$ we conclude that

$$
\lambda\left(V^{\prime}\right) \geq \frac{\lambda\left(\phi\left(V^{\prime}\right)\right)}{L^{2}} \geq \frac{9 \lambda(V)}{16 L^{2}} \geq \frac{9 \lambda(V)}{16 \cdot 81^{2} l^{2}} \geq 10^{-5}(\lambda(V) / \pi) \pi \delta^{2}
$$

which implies that the relative $\lambda$-measure of $g^{-1}(A)$ in any disk $B_{\delta}$ of radius $\delta=$ $\operatorname{diam}\left(V^{\prime}\right)$ centered at any point of $V^{\prime}$ is

$$
\frac{\lambda\left(g^{-1}(A) \cap V^{\prime}\right)}{\lambda\left(V^{\prime}\right)} \cdot \frac{\lambda\left(V^{\prime}\right)}{\lambda\left(B_{\delta}\right)} \geq \frac{10^{-9} \varepsilon e^{-4 \pi m} \lambda(V)}{\pi} .
$$

Since $\lambda(W)=\lambda(\mathbb{D})=\pi$, this concludes the proof.

Lemma 3.2. Let $f$ be a rational map of the sphere and let

$$
B_{f}=\left\{z \in J(f) \mid \omega(z) \backslash A_{f} \neq 0\right\} .
$$

If $R \subset B_{f}$ is such that $\mu(R)>0$, then $R$ is lim sup full.

Proof. Let $R \subset B_{f}$ such that $\mu(R)>0$; then $\lambda(R)>0$, and for the sake of convenience we consider the measure $\lambda$ almost until the end of the proof. Choose a point $z$ of density of $R$ and consider a sequence $\left(n_{i}\right)$ such that $f^{n_{i}}(z) \rightarrow x \notin A_{f}$ (note that then $x \in J(f)$ ). We may assume that $z$ has infinite orbit (there are only countably many points with finite orbits).

Let us show that then we may assume that $x$ is not a parabolic point. Indeed, if $x$ is a periodic point, then it is well known that either its orbit coincides with the entire $\omega(z)$ or $x$ is not isolated in $\omega(z)$. However since any periodic point in the Julia set locally repels points in the Julia set (in case of parabolic points see [CG93, pages 35-41]) we conclude that the former case contradicts the assumption that the orbit of $z$ is infinite. Thus $x$ is not isolated in $\omega(z)$ and we can always pick it so that it is not a parabolic periodic point.

By Theorem 2.3 there exists a round disk $W_{x}=W$ centered at $x$ and a number $N$ such that for any connected component $U$ of $f^{-n}(W)$ the degree of $f^{n} \mid U$ is less than $N$ and, moreover, for any $\varepsilon_{1}>0$ there exists $k$ such that every connected component of $f^{-n}(W)$ has diameter less than $\varepsilon_{1}$ provided that $n>k$. 
Choose now a smaller round disk $V$ with center at $x$ so that $\partial V$ contains no critical value of $f^{n}$ for any $n$ and $\bmod (W \backslash V) \geq 4 \pi N$. Since $f^{n_{i}}(z) \rightarrow x$ we may assume that $f^{n_{i}}(z) \in V$ for any $i$. Then let $U_{i}$ be the component of $f^{-n_{i}}(W)$ containing $z$ and $V_{i}^{\prime} \subset U_{i}$ be the component of $f^{-n_{i}}(V)$ containing $z$.

Let us show that

$$
\limsup \lambda\left(f^{n_{i}}(R) \cap V\right)=\lambda(V) .
$$

Indeed, suppose that this is not the case. Then for some $\varepsilon>0$ and any $i$ the relative measure of $V \backslash f^{n_{i}}(R)$ in $V$ is greater than $\varepsilon$. Apply Lemma 3.1 to $W, V, f^{n_{i}}=g$ and $V \backslash f^{n_{i}}(R)=A$. It implies that the relative measure of the complement to $R$ in the disk of radius $d_{i}=\operatorname{diam}\left(V_{i}\right)$ centered at $z$ is bounded away from 0 . Since $d_{i} \rightarrow 0$ by Theorem 2.3 we get a contradiction with the assumption that $z$ is a point of density of $R$. This contradiction proves that relative $\lambda$-measure of $f^{n_{i}}(R)$ in $V$ has lim sup equal to 1 . Thus the same holds for the measure $\mu$.

Since $V$ is a neighborhood of a point $x \in J(f)$ it contains repelling periodic points of $f$. Choose a repelling periodic point $\zeta \in V$ of period $k$ and its neighborhood $G \subset V$ such that $G \subset f^{k}(G)$. Then the relative $\mu$-measure of $f^{n_{i}}(R)$ in $G$ has lim sup equal to 1 . On the other hand by Montel's Theorem, if $m$ is sufficiently big, then $\mu\left(\widehat{\mathbb{C}} \backslash f^{m k}(G)\right)$ is very small. Therefore, first choosing $m$ big enough (so that $\mu\left(\widehat{\mathbb{C}} \backslash f^{m k}(G)\right)$ is very small) and then sufficiently big $i$ (so that the relative $\mu$-measure of $f^{n_{i}}(R)$ in $G$ is almost equal to 1$)$ we see that the $\mu$-measure of $f^{m k+n_{i}}(R)$ is almost equal to 1 and thus that $R$ is lim sup full. This completes the proof of the lemma.

We can now complete the proof of Theorem 2.1.

Proof. By already known results quoted in the Introduction we may assume that $\mu\left(B_{f}\right)>0$. Since $B_{f}$ is fully invariant, it follows from Lemma 3.2 that $\mu\left(B_{f}\right)=1$. Moreover, if $K$ is a subset of $\widehat{\mathbb{C}}$ such that $\mu(K)>0$, then $\mu\left(K \cap B_{f}\right)>0$ and by Lemma 3.2, $\lim \sup \mu\left(f^{n}\left(K \cap B_{f}\right)\right)=1$. Hence $f$ is lim sup full. By Theorem 2.4, any lim sup full rational map is exact and conservative. It remains to show that $\omega(z)=\widehat{\mathbb{C}}$ for a.e. point of the sphere. To this end it is enough to show that for an open $U$ the set of points which never enter $U$ has zero measure. Indeed, otherwise there exists a set $D$ of positive measure and an open set $U$ such that orbits of $x \in D$ do not enter $U$. But then $\lim \sup _{n \rightarrow \infty} \mu\left(f^{n}(D)\right) \leq 1-\operatorname{area}(U)<1$. This contradicts the fact that $f$ is lim sup full and completes the proof of the theorem.

\section{REFERENCES}

[Bar97] J. A. Barnes, Conservative exact rational maps of the sphere, preprint (1997).

[CG93] L. Carleson and T. W. Gamelin, Complex dynamics, Universitext: Tracts in Mathematics, Springer-Verlag, 1993. MR 94h:30033

[Lyu83] M. Yu. Lyubich, Typical behavior of trajectories of the rational mappings of a sphere, Soviet Math. Dokl. 27:1 (1983), 22-25, Originally published in Dokl. Akad. Nauk SSSR 268:1 (1983), 29-32. MR 84f:30036

[McM94] C. T. McMullen, Complex dynamics and renormalization, Annals of mathematical studies, no. 135, Princeton Univ. Press, 1994. MR 96b:58097

[Mil92] J. Milnor, Locally connected Julia sets: Expository lectures, Tech. Report 11, SUNYStony Brook, 1992, Institute for Mathematical Sciences.

[Mn93] R. Mañé, On a theorem of Fatou, Bol. Soc. Bras. Mat. 24 (1993), 1-11. MR 94g:58188 
1220 ALEXANDER M. BLOKH, JOHN C. MAYER, AND LEX G. OVERSTEEGEN

[Roh64] V. Rohlin, Exact endomorphisms of a Lebesgue space, Amer. Math. Soc. Transl. 39(2) (1964), 1-36.

[Urb94] M. Urbański, Rational functions with no recurrent critical points, Ergodic Th. \& Dyn. Sys. 14 (1994), 391-414. MR 95g:58191

Department of Mathematics, University of Alabama at Birmingham, Birmingham, AlABAMA 35294-1170

E-mail address, Alexander M. Blokh: ablokh@math.uab.edu

E-mail address, John C. Mayer: mayer@math.uab.edu

E-mail address, Lex G. Oversteegen: overstee@math.uab.edu 